\title{
Physiological traits of invertebrates entering cryptobiosis in a post-embryonic stage
}

\author{
Masahiko WATANABE ${ }^{1}$, Takahiro KIKAWADA ${ }^{1}$, AkihiKo FUJITA ${ }^{1}$, Ewa FORCZEK ${ }^{2}$, Taro ADATI ${ }^{3,4}$ \\ and TAKASHI OKUDA ${ }^{1 *}$ \\ ${ }^{1}$ National Institute of Agrobiological Sciences, Ohwashi 1-2, Tsukuba, Ibaraki 305-8634, Japan \\ ${ }^{2}$ Jagiellonian University, Ingardena 6, 30-060 Krakow, Poland \\ ${ }^{3}$ International Institute of Tropical Agriculture (Kano Station), Sabo Bakin Zuwo Road, P.M.B. 3112, Kano, Nigeria \\ ${ }^{4}$ Present address: Tokyo University of Agriculture, Tokyo 156-8502, Japan
}

Key words. Cryptobiosis, anhydrobiosis, invertebrate, post-embryonic stage, trehalose, compatible solute, recovery

\begin{abstract}
Cryptobiosis is the state when the metabolic activity of an organism is hardly measurable or is reversibly at a standstill. Many groups of invertebrates have this ability, and can be divided into two types according to the developmental stage in which it occurs; embryonic (eggs) or post-embryonic stages (larvae and adults). The latter must be able to reversibly regulate the physiology and biochemistry of development and cryptobiosis. There are several reviews on cryptobiosis and its regulation, but none on the physiological mechanism of cryptobiosis in chironomids. The present paper reviews the physiological traits of invertebrates entering cryptobiosis in a post-embryonic stage. These unique phenomena, which occur in a post-embryonic stage of three groups of cryptobiotic invertebrates (insects, tardigrades and nematodes) are discussed with particular reference to; 1) the behavioural and physiological adaptations of cryptobiotic invertebrates, 2) role of trehalose in cryptobiosis and 3) regulation of cryptobiosis.
\end{abstract}

\section{INTRODUCTION}

In 1702, Leeuwenhoek observed inactive animalcules (tardigrades or rotifers) of an oval shape in the dry sediments in the gutters of roofs of houses. The animalcules started moving shortly after coming into contact with water. Such a phenomenon is termed cryptobiosis, and is the state in an organism when its metabolic activity is hardly measurable, or reversibly at a standstill (Keilin, 1959).

Keilin (1959) noted that cryptobiosis could be divided into several categories; cryobiosis (freezing), osmobiosis (extremely high level of solutes), anoxybiosis (lack of oxygen) and anhydrobiosis (desiccation). The last category, anhydrobiosis, is common and found in many taxa ranging from unicellular organisms to higher invertebrates and plants.

Cryptobiosis is found in many groups of invertebrates including insects (chironomids), tardigrades, nematodes, rotifers, collembolans and primitive crustaceans (brine shrimp, Artemia) (Sømme, 1995). Cryptobiotic organisms can be divided into two types according to the developmental stage in which cryptobiosis occurs; embryonic or post-embryonic. In the former, as in Artemia and collembola, female parents prepare the molecules necessary for cryptobiosis in their progenies (eggs) to some extent in response to external conditions (Hochachka \& Guppy, 1987). In contrast, in the latter case, as in chironomids, most tardigrades, nematodes and rotifers, parents do not affect induction of cryptobiosis in their progenies at all, and individuals themselves must be able to reversibly switch their physiology and biochemistry between devel- opment and cryptobiosis. That is, in this case more complex physiological mechanisms are associated with cryptobiosis.

An African chironomid, Polypedilum vanderplanki, is the most advanced and largest multicellular animal with cryptobiotic ability in a post-embryonic stage. Hinton reported extremely high tolerance of various stresses by larvae of this chironomid between 1951 and 1968 (Hinton, 1951, 1960a, b, 1968), but the mechanisms of cryptobiosis in this unique chironomid have not been investigated. In 2000, we successfully reared this chironomid continuously in the laboratory and started to use it to study the physiology of cryptobiosis.

There are reviews on cryptobiosis and its regulation (Hochachka \& Guppy, 1987; Crowe et al., 1992; Sømme, 1995; Clegg, 2001). However, they do not include an account of the physiological mechanism of chironomid cryptobiosis. The present paper reviews the physiological traits of invertebrates that enter cryptobiosis in a postembryonic stage, including the recently obtained data on chironomids. We mention and discuss this unique phenomena in the three main groups of invertebrates (insects, tardigrades and nematodes), which enter cryptobiosis in a post-embryonic stage, in particular; 1) the behavioural and physiological adaptations of cryptobiotic invertebrates, 2) role of trehalose in cryptobiosis and 3) regulation of cryptobiosis.

\section{CHIRONOMID}

P. vanderplanki, is the most advanced and largest multicellular cryptobiotic invertebrate (length of larvae 6-7

\footnotetext{
* Corresponding author; e-mail: oku@affrc.go.jp
} 


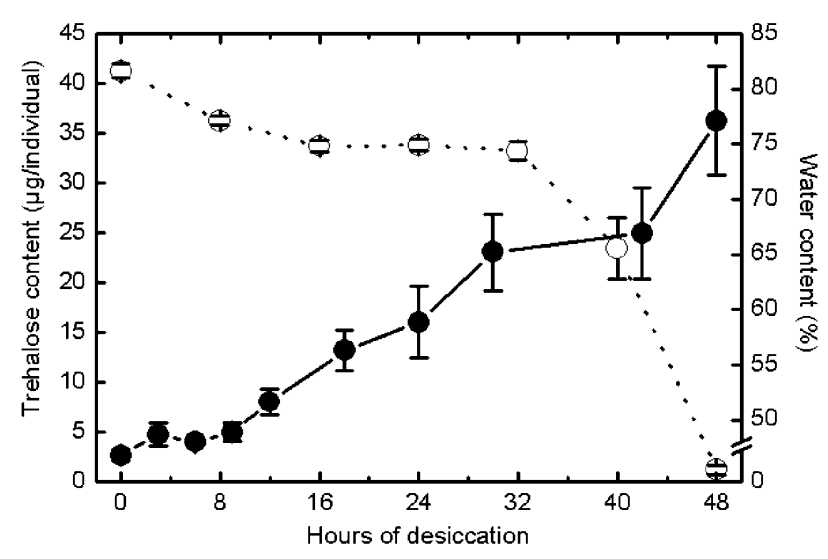

Fig. 1. Changes in the trehalose and water content of $P$. vanderplanki larvae during desiccation over a period of 2 days. Solid line with closed symbols, trehalose content; dotted line with open symbols, water content. Each point is a mean \pm SE. N $=8-10$ for trehalose and 12 for water. Data from Watanabe et al. (2003).

$\mathrm{mm})$. This species breeds in small temporary pools formed in shallow hollows in unshaded rocks in Nigeria and Uganda (Hinton, 1951, 1960a). The cryptobiotic larvae show an extremely high thermal tolerance from -270 to $103^{\circ} \mathrm{C}$, and can revive and grow after submersion in pure ethanol or glycerol (Hinton, 1960a, b, 1968). The longest record of dry preservation for this species is 17 years (Adams, 1985).

In the field, larvae usually live in soil nest tubes in pools (Hinton, 1951). When the pools dry up, the larvae gradually dry out and fold in the middle. The soil nest tubes decrease the rate of loss of water from the larvae. However, the folded larval form is not essential for inducing cryptobiosis in this insect; larvae that were induced to enter cryptobiosis on wet filter paper were often unfolded (Watanabe et al., 2002).

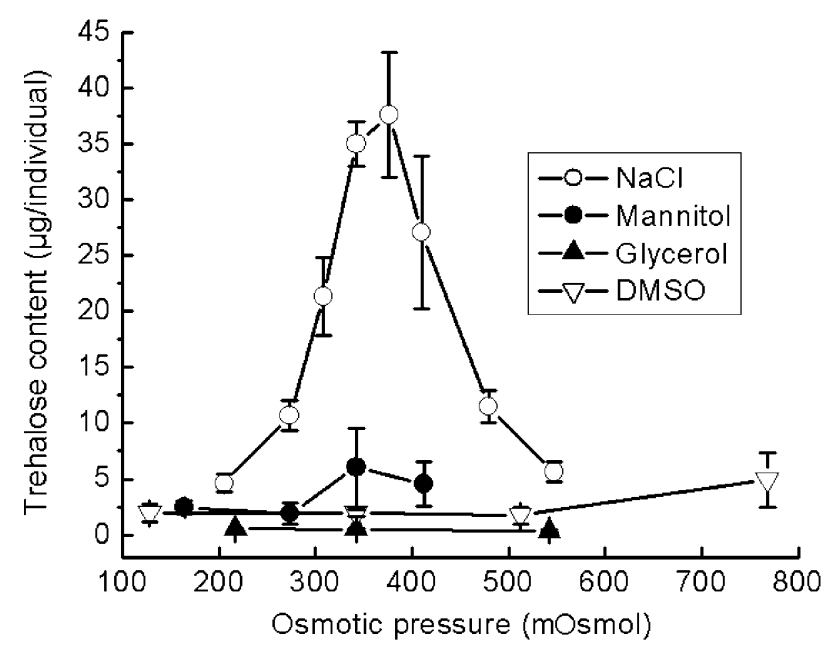

Fig. 2. Trehalose content of $P$. vanderplanki larvae incubated for 1 day in $\mathrm{NaCl}$, mannitol, glycerol and DMSO solutions of various osmotic pressures. One \% of $\mathrm{NaCl}$ solution is $342 \mathrm{mOs}$. Each point is a mean \pm SE. $N=4-10$ each. Data from Watanabe et al. (2003).

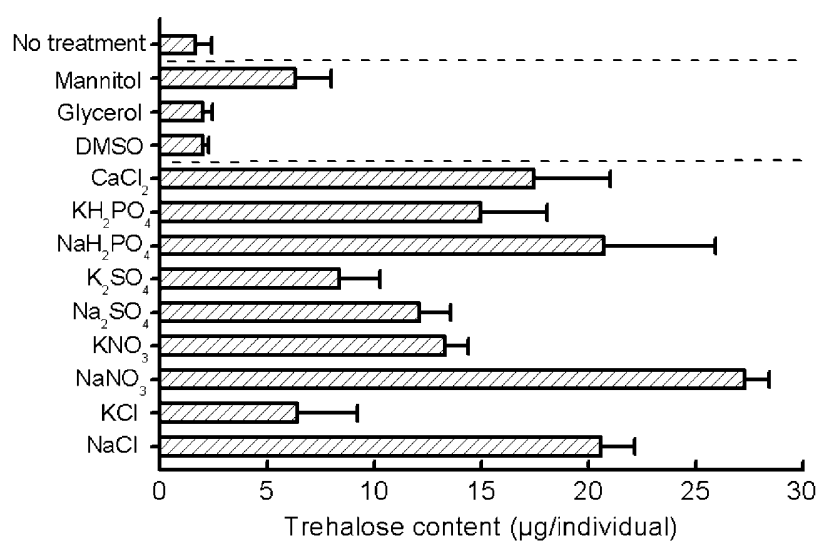

Fig. 3. Effect of various salt solutions on the trehalose content of $P$. vanderplanki larvae. Larvae were incubated for 1 day in each solution, which all had the same osmotic pressure (342 mOs). Control shows the trehalose content of untreated larvae ("no treatment"). Each column shows mean $\pm \mathrm{SE} . \mathrm{N}=5-8$ each. Data from Watanabe et al. (2003).

Larvae in water usually contain a small amount of trehalose in their blood. During the process of desiccation, larvae accumulate trehalose to around $20 \%$ of the dry body weight (Fig. 1). Other sugars and polyols were not detected. On the other hand, larvae dehydrated within 1 day do not accumulate as much trehalose and do not recover after rehydration (Watanabe et al., 2003).

Watanabe et al. (2003) demonstrated that the increase in the internal ion concentration triggers the rapid trehalose synthesis in $P$. vanderplanki. Trehalose accumulates in larvae kept in highly concentrated salt solutions (Fig. 2) depending on ion in solution (Fig. 3). It does not accumulate when larvae are kept in solutions of DMSO or glycerol. The yeast, Saccharomyces cerevisiae, has an osmosensor that activates glycerol synthesis in response to high osmolarity regardless of solute (Maeda et al., 1994; Posas \& Saito, 1997; Maeda, 1999). Unlike in yeast

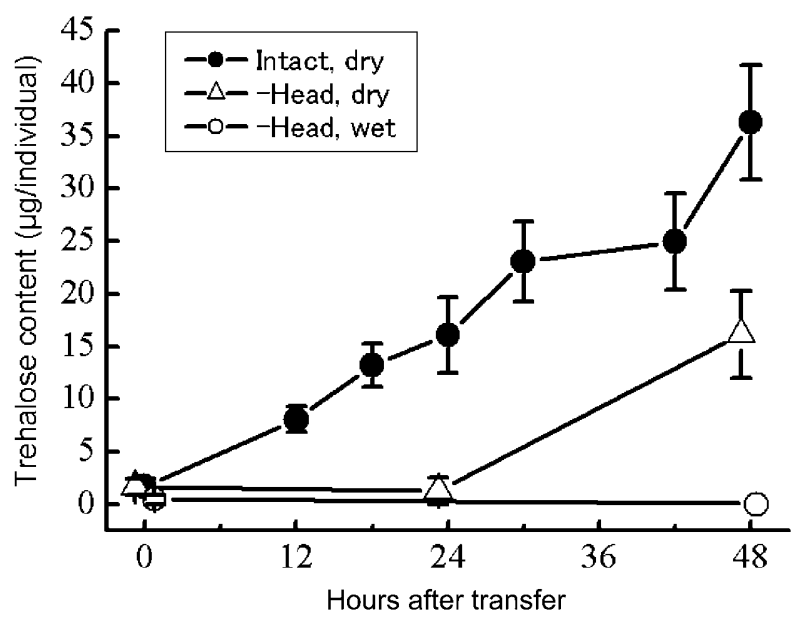

Fig. 4. Change in the trehalose content of $P$. vanderplanki larvae during desiccation over a period of 2 days. Intact, dry desiccated intact larvae (closed circles); head, dry - desiccated decapitated larvae (open triangles); head, wet - decapitated larvae in water (open circles). Each point is a mean $\pm \mathrm{SE} . \mathrm{N}=$ 3-9. Data from Watanabe et al. (2002). 

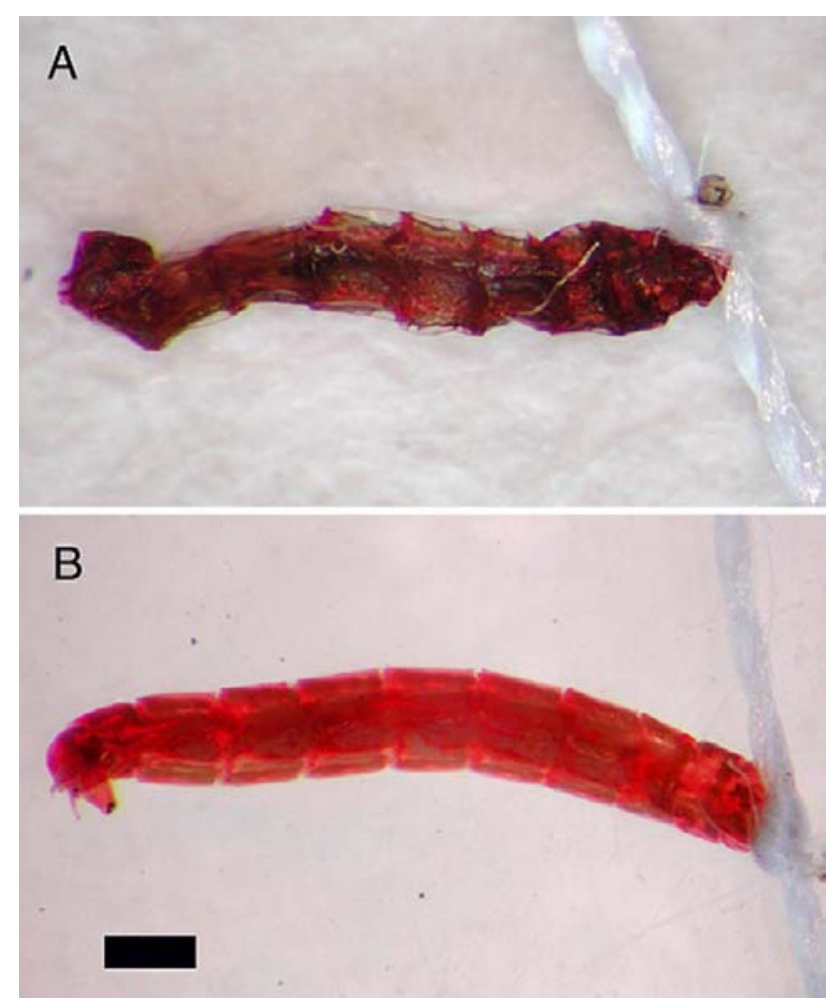

Fig. 5. Recovery from cryptobiosis of decapitated larvae of $P$. vanderplanki. A - decapitated cryptobiotic larva. B - decapitated larva on the second day after rehydration. Photographs from Watanabe et al. (2002).

the rapid accumulation of trehalose in $P$. vanderplanki larvae is not a simple osmotic response.

Many temperate insects enter diapause in various developmental stages in order to survive adverse conditions. Although the endocrines regulating larval, pupal and adult diapause differ, the brain is the common prime regulator of diapause (Denlinger, 1985). Watanabe et al. (2002) have demonstrated that cryptobiosis can occur in $P$. vanderplanki larvae without a brain. Larvae without a head accumulate relatively large amounts of trehalose during desiccation (Fig. 4) and recover after rehydration like intact larvae (Fig. 5). Furthermore, we recently found that an isolated fat body can synthesize a large amount of trehalose during desiccation and after desiccation for a week can recover on rehydration (Watanabe et al., unpubl. data). Therefore, it is likely that the central nervous system is not involved in the induction of cryptobiosis in this chironomid.

\section{TARDIGRADES}

There are 600 species of Tardigrada in the world (Pilato, 1979). They usually live in fresh water or humid soil. Anhydrobiosis is widespread in this phylum and occurs mainly in the adult stage.

There is an abundance of literature on anhydrobiosis in tardigrades covering more than 200 years, which has been reviewed by Ushatinskaya (1990), Wright et al. (1992), Sømme $(1995,1996)$. Spallanzani (1769) showed that tar- digrades could survive being kept in a vacuum. In the anhydrobiotic state, tardigrades can tolerate extreme temperatures from $-270^{\circ} \mathrm{C}$ to $151^{\circ} \mathrm{C}$, exposure to X-rays $(570,000 \mathrm{R})$ and high hydrostatic pressure $(600 \mathrm{Mpa})$ (Rahm, 1937; Crowe \& Cooper, 1971; Seki \& Toyoshima, 1998). The revival rate gradually decreases with increase in the dehydration period; the maximum period for which anhydrobiotic Anguillulina tritici can survive may be 10 years (Keilin, 1959).

Anhydrobiotic tardigrades always contract into a structure resembling a small tun when dehydrated (Sømme, 1995). Tun formation is essential for the successful induction of anhydrobiosis in tardigrades. The rates of water loss and transpiration gradually decrease as the surface area is reduced during tun formation (Wright et al., 1992). Both rates rapidly decline just after completion of tun formation, finally to an undetectable level. When tardigrades are desiccated at a low relative humidity or under anoxia they cannot form tun and be revived (Crowe, 1972).

Trehalose accumulation is also observed in the tardigrade, Adorybiotus coronifer, during dehydration (Westh \& Ramløv, 1991). Trehalose in active individuals of this species makes up $0.1 \%$ of the dry body weight. During dehydration, this tardigrade completes tun formation in $3 \mathrm{~h}$ and accumulates trehalose up to $2.3 \%$ of the dry weight within $7 \mathrm{~h}$. The level of trehalose accumulation is much lower than that often found in other anhydrobiotic organisms (10-20\%) (Sømme, 1995; Clegg, 2001).

Protein synthesis resumes $2 \mathrm{~h}$ after rehydration and the trehalose content decreases to the normal level $6 \mathrm{~h}$ after rehydration (Westh \& Ramløv, 1991). $\mathrm{CO}_{2}$ induction of aerobic acidosis in rehydrating tardigrades caused a reduction in anabolic and catabolic activities, trehalose degradation and protein synthesis.

\section{NEMATODES}

Most anhydrobiotic animals are nematodes (Womersley, 1987). A number of anhydrobiotic nematodes are widely distributed from deserts to the Antarctic area (Ellenby 1969; Crowe \& Madin, 1975; Womersley, 1987). Anhydrobiosis in nematodes occurs in juvenile and adult stages, but sometimes in the egg cysts within protective shells (Sømme, 1995). Cryptobiotic nematodes can be revived after exposure to extremely low temperatures (Pickup \& Rothery, 1991; Wharton \& Barclay, 1993). Anguina tritici and Tylenchus polyhypnus recovered from the anhydrobiotic state after 32 and 39 years, respectively (Steiner \& Albin, 1946; Womersley, 1980).

Anhydrobiotic nematodes can be divided into two groups; slow-dehydration and fast-dehydration strategists (Womersley, 1987; Wharton, 2002). The former need to be dehydrated slowly at high relative humidity (RH) to successfully enter anhydrobiosis. On the other hand, the fast-dehydration strategists can survive relatively high rates of dehydration or possess adaptations that slow down the rate of water loss.

Slow rate of water loss is achieved by behavioural and/or physiological mechanisms (Wharton, 2002). The 
most common behavioural strategies for decreasing the rate of water loss are coiling and clumping. Coiling in Aphelenchus avenae decreases the rate of water loss by decreasing the external surface of the body, enabling these nematodes to enter cryptobiosis (Crowe \& Madin, 1975). Clumping (forming of an aggregation) by nematodes also decreases water loss, especially of the nematodes in the center of the aggregation, and increases their survival in the desiccated state (Ellenby, 1969).

A physiological strategy for decreasing the rate of water loss is to change the permeability of the body surface (Wharton, 2002). Ditylenchus dipsaci decreases water loss by rapidly lowering body surface permeability 2 min after the onset of desiccation (Wharton, 1996). This occurs via a phase change in the composition of the epicuticle or a decrease in the thickness of the cuticle (Wharton \& Lemmon, 1998).

Many species of nematodes accumulate low-molecular weight carbohydrates in the anhydrobiotic phase (Womersley, 1987). However, the nature and content of these compounds depends upon nematode; larvae of Ap. avenae accumulated trehalose (11-13\% of the dry weight) and glycerol (5\%) (Madin \& Crowe, 1975). An. tritici increased its inositol $(0.8 \%)$ and trehalose $(9 \%)$ content during dehydration (Womersley \& Smith, 1981). On the other hand, Higa \& Womersley (1993) demonstrated that the accumulation of trehalose in itself is not sufficient for the induction of anhydrobiosis in Ap. avenae; the nematode survival was directly related to rates of evaporative water loss rather than trehalose content. Other physiological and biochemical adaptations for surviving anhydrobiosis may exist in nematodes (Womersley, 1987).

\section{IMPORTANCE OF TREHALOSE IN DEHYDRATION}

Trehalose is a common compatible solute in cryptobiotic organisms, such as unicellular organisms (bacteria, yeast and spores of fungus), invertebrates (chironomid, tardigrades, nematodes and encysted Artemia) and resurrection plants, although other disaccharides such as sucrose is present in seeds and pollen grains of higher plants (Ingram \& Bartels, 1996; Clegg, 2001). Most of these organisms accumulate quite a large amount of trehalose, around $10-20 \%$ of the dry body weight.

It is of interest to discuss why trehalose is the compatible solute in cryptobiotic organisms ranging from unicellular organisms to higher invertebrates and plants. Trehalose, as a non-reducing sugar, is less harmful to cells and tissues than reducing sugars such as glucose even at extremely high concentrations. Among sugars and polyols trehalose provides the most effective protection against desiccation because of its high ability for waterreplacement and glass formation (vitrification) (Burke, 1986; Crowe et al., 1987; Green \& Angell, 1989; Crowe et al., 1998); it substitutes for bound and free water, and so maintains the structures of cell membranes and proteins. Glassy state of trehalose may fill the spaces in tissues during dehydration and allow the orderly packing of body components, which prevents structural damage, and inhibits aggregation of biological molecules and increase in solute concentration. High and stable viscosity of trehalose glasses may also stop all chemical reactions that require molecular diffusion.

Recently it has been shown that human primary fibroblasts intracellularly expressing trehalose, due to a recombinant adenovirus vector, could be maintained in a completely dry state for a short period (Guo et al., 2000). Freeze-dried human and mammalian platelets can recover after rehydration only when they were loaded intracellularly with a relatively high concentration of trehalose before freeze-drying (Wolker et al., 2001, 2002). This indicates that the presence of intracellular trehalose might be important for desiccation tolerance. The study of the localisation of intra- and extra-cellular trehalose in anhydrobiotic invertebrates may provide important information for elucidating the mechanism of cryptobiosis.

\section{CONCLUSIONS}

The post-embryonic stages of many species of invertebrates survive complete dehydration by entering cryptobiosis. Such invertebrates have evolved behavioural and physiological adaptations for the successful induction of cryptobiosis. Slow desiccation is necessary for cryptobiosis in most cases. The most common physiological feature is the accumulation of trehalose, although anhydrobiotic rotifers, Philodina roseola and Adineta vaga, do not have trehalose and any other low-molecular weight carbohydrates during dehydration (Lapinski \& Tunnacliffe, 2003). However, the existence of a large amount of trehalose in itself is not sufficient for anhydrobiosis in nematodes and a chironomid (Higa \& Womersley, 1993; Watanabe et al., 2003). This may indicate that factor(s) other than trehalose are involved in regulating cryptobiosis. In plants, chemicals such as prolin, betaine and the late embryogenesis abundant (LEA) protein, as well as sugars are thought to protect cells against desiccation (Yamaguchi-Shinozaki, 2003). In fact, the anhydrobiotic nematod, Ap. avenae has LEA protein gene (Browne et al., 2002), and the anhydrobiotic rotifers, $P$. roseola and $A d$. vaga, have a dehydration-regulated protein which is recognized by an antiserum against a nematode LEA protein (Tunnacliffe et al., 2004).

The carbon source for the solutes that protect against desiccation is uniformly distributed in unicellular organisms and plants, i.e., mainly glucose in the former and sucrose in the latter (Ingram \& Bartels, 1996). By contrast, in insects, glycogen is the main source, and it is stored in the fat body (Storey \& Storey, 1991). P. vanderplanki larvae have a large amount of glycogen in their fat body at hydrated state and of trehalose in their hemolymph just prior to complete dehydration (Watanabe et al., unpubl. data). It is likely that the distribution of newly synthesized trehalose to all cells and tissues, probably from the fat body via the hemolymph, is important for dehydrating larvae. Invertebrates that enter cryptobiosis in a post-embryonic stage may have more complex physiological and molecular mechanisms for inducing of cryptobiosis than cryptobiotic unicellular organisms and plants. 


\section{REFERENCES}

Adams S. 1985: Cryptobiosis in Chironomidae (Diptera) - two decades on. Antenna 8: 58-61.

Browne J., Tunnacliffe A. \& Burnell A. 2002: Plant desiccation gene found in a nematode. Nature 416: 38 .

BuRKE M.J. 1986: The glassy state and survival of anhydrous biological systems. In Leopold A.C. (ed.): Membranes, Metabolism, and Dry Organisms. Comstock Publishing Associate, Cornell University Press, Ithaca and London, pp. 358-363.

ClegG J.S. 2001: Cryptobiosis - a peculiar state of biological organization. Comp. Biochem. Physiol. (B) 128: 613-624.

Crowe J.H. 1972: Evaporative water loss by tardigrades under controlled relative humidities. Biol. Bull. 142: 407-416.

Crowe J.H. \& Cooper A.F. 1971: Cryptobiosis. Sci. Am. 225: 30-36.

Crowe J.H. \& MadiN K.A.C. 1975: Anhydrobiosis in nematodes: evaporative water loss and survival. J. Exp. Zool. 193: 323-334.

Crowe J.H., Crowe L.M., Carpenter J.F. \& Wistrom C.A. 1987: Stabilization of dry phospholipid bilayers and proteins by sugars. Biochem. J. 242: 1-10.

Crowe J.H., Hoekstra F.A. \& Crowe L.M. 1992: Anhydrobiosis. Annu. Rev. Physiol. 54: 579-599.

Crowe J.H., Carpenter J.F. \& Crowe L.M. 1998: The role of vitrification in anhydrobiosis. Annu. Rev. Physiol. 60: 73-103.

DenLINGER D.L. 1985: Hormonal control of diapause. In Kerkut G.A. \& Gilbert L. (eds): Comprehensive Insect Physiology, Biochemistry and Pharmacology. Vol. 8. Endocrinology II. Pergamon Press, Oxford, pp. 353-412.

Ellenby C. 1969: Dormancy and survival in nematodes. Soc. Exp. Biol. Symp. 23: 83-97.

Green J.L. \& Angell C.A. 1989: Phase relations and vitrification in saccharide-water solutions and the trehalose anomaly. J. Phys. Chem. 93: 2880-2882.

Guo N., Puhlev I., Brown D.R., Mansbridge J. \& Levine F. 2000: Trehalose expression confers desiccation tolerance on human cells. Nature Biotech. 18: 168-171.

Higa L.M. \& WomersLey C. 1993: New insights into the anhydrobiotic phenomenon - effects of trehalose content and differential rates of evaporative water loss on the survival of Aphelenchus avenae. J. Exp. Zool. 267: 120-129.

HinTon H.E. 1951: A new chironomid from Africa, the larva of which can be dehydrated without injury. Proc. Zool. Soc. Lond. 121: 371-380.

Hinton H.E. 1960a: Cryptobiosis in the larva of Polypedilum vanderplanki Hint. (Chironomidae). J. Insect Physiol. 5: 286-300.

Hinton H.E. 1960b: A fly larva that tolerates dehydration and temperatures of $-270^{\circ}$ to $+102^{\circ} \mathrm{C}$. Nature 188: $336-337$.

Hinton H.E. 1968: Reversible suspension of metabolism and the origin of life. Proc. R. Soc. (B) 171: 43-57.

Hochachka D.W. \& Guppy M. 1987: Metabolic Arrest and the Control of Biological Time. Harvard University Press, Cambridge, Massachusetts and London, $227 \mathrm{pp}$.

IngRAM J. \& BARTELS D. 1996: The molecular basis of dehydration tolerance in plants. Annu. Rev. Plant Physiol. Plant Mol. Biol. 47: 377-403.

KeILIN D. 1959: The problem of anabiosis or latent life history and current concept. Proc. R. Soc. London (B) 150: 149-191.

LAPINSKi J. \& TunNaClifFe A. 2003: Anhydrobiosis without trehalose in bdelloid rotifers. FEBS Lett. 553: 387-390.
Madin K.C. \& CRowe J.H. 1975: Anhydrobiosis in nematodes: carbohydrate and lipid metabolism during dehydration. $J$. Exp. Zool. 193: 335-342.

MAEDA T. 1999: Osmotic response of the yeast Saccharomyces cerevisiae. In Shinozaki K., Yamamoto M., Okamoto H. \& Iwabuchi M. (eds): Molecular Mechanisms of Response and Adaptation to Environmental Stimuli. (Special issue of Protein, Nucleic acid, Enzyme). Kyoritsu Publishing, Tokyo, pp. 2206-2213 (in Japanese).

Maeda T., Wurgler-Murphy S.M. \& Saito H. 1994: A twocomponent system that regulates an osmosensing MAP kinase cascade in yeast. Nature 369: 242-245.

Pickup J. \& Rothery P. 1991: Water loss and anhydrobiotic survival in nematodes from Antarctic fell fields. Oikos 61: 379-388.

Pilato G. 1979: Correlations between cryptobiosis and other biological characteristics in some soil animals. Bull. Zool. 46: 319-332.

Posas F. \& SaIto H. 1997: Osmotic activation of the HOG MAPK pathway via Ste11p MAPKKK: scaffold role of Pbs2p MAPKK. Science 276: 1702-1705.

RAHм G. 1937: A new ordo of tardigrades from the hot springs of Japan (Furu-yu section, Unzen). Ann. Zool. Jpn. 16: 345-352.

Seki K. \& Toyoshima M. 1998: Preserving tardigrades under pressure. Nature 395: 853-854.

Søмme L. 1995: Invertebrates in Hot and Cold Arid Environments. Springer-Verlag, Germany, $280 \mathrm{pp}$.

Søмme L. 1996: Anhydrobiosis and cold tolerance in tardigrades. Eur. J. Entomol. 93: 349-357.

Spallanzani L. 1769: Nouvelles Recherches sur les Découvertes Microscopique et sur la Génération des Corps Organiques. Vol. 1 .

STeiner G. \& Albin F.E. 1946: Resuscitation of the nematode Tylenchus polyhypnus, n. sp., after almost 39 years' dormancy. J. Wash. Acad. Sci. 36: 97-99.

Storey K.B. \& Storey J.M. 1991: Biochemistry of cryoprotectants. In Lee R.E. \& Denlinger D.L. (eds): Insects at Low Temperature. Chapman and Hall, New York and London, pp. 64-93.

Tunnacliffe A., Lapinski J. \& McGee B. 2004: Hydrobiologia, in press.

Ushatinskaya R.S. 1990: Latent Life and Anabiosis. Nauka, Moscow, 183 pp. (in Russian).

Watanabe M., Kikawada T., Minagawa N., Yukuhiro F. \& OKUDA T. 2002: Mechanism allowing an insect to survive complete dehydration and extreme temperatures. J. Exp. Biol. 205: 2799-2802.

Watanabe M., KiKawada T. \& OKuda T. 2003: Increase of internal ion concentration triggers trehalose synthesis associated with cryptobiosis in larvae of Polypedilum vanderplanki. J. Exp. Biol. 206: 2281-2286.

Westh P. \& Ramløv H. 1991: Trehalose accumulation in the tardigrades Adorybiotus coronifer during anhydrobiosis. $J$. Exp. Zool. 258: 303-311.

WhARTON D.A. 1996: Water loss and morphological changes during desiccation of the anhydrobiotic nematode Ditylenchus dipsaci. J. Exp. Biol. 199: 1085-1093.

WharTon D.A. 2002: Nematode survival strategies. In Lee D.L. (ed.): The Biology of Nematodes. Taylor \& Francis, London, pp. 389-411.

Wharton D.A. \& Barclay S. 1993: Anhydrobiosis in the freeliving Antarctic nematode, Panagrolainus davidi (Nematoda: Rhabditidae). Fundam. Appl. Nematol. 16: 17-22. 
Wharton D.A. \& Lemmon J. 1998: Ultrastructural changes during desiccation of the anhydrobiotic nematode Ditylenchus dipsaci. Tissue Cell 30: 312-323.

Wolkers W.F., Tablin F. \& Crowe J.H. 2002: From anhydrobiosis to freeze-drying of eukaryotic cells. Comp. Biochem. Physiol. (A) 131: 535-543.

Wolkers W.F., Walker N.J., Tablin F. \& Crowe J.H. 2001: Human platelets loaded with trehalose survive freezingdrying. Cryobiology 42: 79-87.

WomERSLEY C. 1980: The effect of different periods of dehydration/rehydration periods upon the ability of second stage larvae of Anguina tritici to survive desiccation at $0 \%$ relative humidity. Ann. Appl. Biol. 95: 221-224.
WomersLey C. 1987: A reevaluation of strategies employed by nematode anhydrobiotes in relation to their natural environment. In Veech J.A. \& Dickson D.W. (eds): Vistas on Nematology. Society of Nematologists, Hyattsville, MD, pp. 165-173.

Womersley C. \& Smith L. 1981: Anhydrobiosis in nematodes I. the role of glycerol, myo-inositol and trehalose during desiccation. Comp. Biochem. Physiol. (B) 70: 579-586.

Wright J.C., Westh P. \& Ramløv H. 1992: Cryptobiosis in tardigrada. Biol. Rev. 67: 1-29.

YAMAGUCHI-SHINOZAKI K. 2003: Molecular mechanisms of plant responses and tolerance of drought and cold stresses. Cryobiol. Cryotech. 49: 21-27 (in Japanese).

Received October 15, 2003; revised March 15, 2004; accepted June 8, 2004 